\title{
VIBRATION AMPLIFICATION BY REPETITIVE PULSING OF RAPIDLY HEATED CYLINDERS
}

\author{
Robert J. GROSSMAN and Louis J. HAMILTON \\ Department of Nuclear Engineering, The University of Michigan, \\ Ann Arbor, Michigan 48105, U.S.A.
}

Received 9 March 1972

\begin{abstract}
The fuel elements of a repetitively pulsed fast reactor are subjected to rapid heating in a time short with respect to their mechanical response time. As a result, vibration of the fuel elements occurs and, under certain conditions, these vibrations may be amplified considerably.

This work is an experimental investigation of the amplification which occurs when a thin wall, hollow, stainless steel cylinder is repetitively heated internally by Joule heating. The results, which compare well with a theoretical analysis of this problem performed by another author, show that significant amplification of the vibrations can occur.

The experimental technique is a variation of the resonance method for measuring natural frequency and internal damping. The apparatus could be adapted for use as a materials testing facility for high cycle, low stress fatigue studies since relatively large stresses could be induced with a rather small energy deposition per pulse.
\end{abstract}

\section{Introduction}

Under the rapid heating conditions in repetitively pulsed fast reactor fuel elements, significant axial vibrations result from the inability of the fuel elements to expand as quickly as they are heated, due to their inertia [1]. Although the resulting stresses may not be great enough to cause damage to the fuel or surrounding support structure, the number of stress cycles may be very large, since for each reactivity pulse, a number of stress cycles will occur, depending on the available damping mechanisms. The SORA reactor has been designed with a pulse repetition rate of $50 \mathrm{pps}$ [2]. If each reactivity pulse yields 10 stress cycles, the number of stress cycles will approach $10^{8}$ $10^{9}$ in just $2-3$ months of steady operation.

The two primary mechanisms for dissipation of the vibrational energy are: (1) impedance mismatching through contact areas with the cladding and other components, (2) internal friction. Normally the first of these is sufficient to damp out the oscillations due to one pulse before the next pulse occurs. However, as the fuel residence time increases, deformations in the fuel slugs may cause loss of contact areas. The internal friction of some of the metallic fuels proposed for these reactors is quite small; thus, the fuel slug vibration may not damp out within one pulsing period. In this case the displacement cannot be taken as due to a single pulse but must be viewed as the superposition of displacements due to all previous pulses. Consequently, significant amplification of the displacement is a distinct possibility.

This possibility has been studied analy tically by Randles [2], who gives an expression for the asymptotic axial displacement amplification as a function of the fuel element natural frequency of oscillation, the fuel internal damping constant, and the pulsing period. For a given fuel material, Randles shows that the amplification can be quite large if the pulsing period is very close to, if not exactly, an integer multiple of the fundamental period of oscillation.

This paper describes an experimental investigation of vibration amplification. A thin wall, hollow, stainless steel cylinder was repetitively heated by discharging a capacitor through a low inductance circuit containing the sample. High frequency pulsing was accomplished using a triggered spark gap switch. The resulting displacements were measured with a laser interferometer. 
The measured maximum amplification was approximately $60 \%$ of that predicted the oretically by Randles, while the experimental amplification as a function of the pulsing period agreed well with the theory. The results demonstrate that relatively large displacements may be obtained with a rather small energy deposition per pulse. Repetitive internal heating could be used for high cycle fatigue studies, and also as a form of the resonance technique for determining the sample natural frequency as well as the material relaxation time.

\section{Theoretical analysis}

The analysis of Randles is summarized here. A thin, long rod of length $2 h$ is subjected to a uniform step rise in temperature, $T$. The ends are assumed to be free and there is no damping. Randles [3] showed that the longitudinal displacement, $u$, is given by

$$
\begin{aligned}
u(z, t) & =-\alpha h T \frac{8}{\pi^{2}} \sum_{n=0}^{\infty} \frac{(-1)^{n}}{(2 n+1)^{2}} \\
& \times \sin \left((2 n+1) \frac{\pi z}{2 h}\right) \cos \left((2 n+1) \frac{\pi a t}{2 h}\right),
\end{aligned}
$$

where $\alpha$ is the linear thermal expansion coefficient, $a$ is the acoustic velocity, and $z=0$ at the mid-point of the rod. Energy dissipation by internal friction and heat conduction is incorporated into this expression, using first order perturbarion theory, to yield

$$
\begin{aligned}
u(z, t) & =-\alpha h T \frac{8}{\pi^{2}} \sum_{n=0}^{\infty} \frac{(-1)^{n} \exp \left(-t / \tau_{n}\right)}{(2 n+1)^{2}} \\
& \times \sin \left[(2 n+1) \frac{\pi z}{2 h}\right] \cos \left[(2 n+1) \frac{\pi a t}{2 h}\right],
\end{aligned}
$$

where $\tau_{n}$ is the damping time of the $n$th mode. The fundamental term $(n=0)$ of eq. (1) accounts for $8 / \pi^{2}$ $(81 \%)$ of the total displacement. In addition, from eq. (2), each mode damps independently and Randles shows that the damping increases very rapidly with mode number. Thus for $t>\tau_{1}$, essentially only the fundamental mode remains and the assumption is made that the complete displacement description can be approximated by the fundamental mode. Thus,

$$
u(z, t)=d \exp \left(-t / \tau_{\mathrm{r}}\right) \sin k z \cos \omega t,
$$

where $d=-\left(8 / \pi^{2}\right) \alpha h T, k=\pi / 2 h, \omega=\pi a / 2 h$, and $\tau_{\mathrm{r}}=\tau_{0}$.

The assumption is then made that a uniform step rise in temperature, $T$, occurs at repeated intervals, $p$. By superposition, the longitudinal displacement of the rod after the $(m+1)$ st pulse is given by

$$
\begin{aligned}
u_{m+1} & =A_{m+1} d \exp \left[-(t \cdots m p) / \tau_{\mathrm{r}}\right] \\
& \times \sin k z \cos \left(\omega t-\Theta_{m+1}\right)
\end{aligned}
$$

where $A_{m+1}$ is the amplitude amplification factor and $\Theta_{m+1}$ is the phase shift for the $(m+1)$ st pulse. These are given by the recurrence relations

$$
\begin{aligned}
A_{m+1} & =\left[\left(\xi A_{m} \cos \Theta_{m}+\cos m \omega p\right)^{2}\right. \\
& \left.+\left(\xi A_{m} \sin \Theta_{m}+\sin m \omega p\right)^{2}\right]^{\frac{1}{2}}, \\
\Theta_{m+1} & =\tan ^{-1}\left(\frac{\xi A_{m} \sin \Theta_{m}+\sin m \omega p}{\xi A_{m} \cos \Theta_{m}+\cos m \omega p}\right),
\end{aligned}
$$

where $\xi=\exp \left(-p / \tau_{\mathrm{r}}\right)$.

As the number of pulses increases, the ratio of $A_{m+1} / A_{m}$ decreases and the displacement amplification approaches an asymptotic value, $A$. In order for eq. (5) to be valid, when $A_{m} \approx A_{m+1}=A$.

$$
\Theta_{m}=\Theta+m \omega p
$$

where $\Theta$ is a constant which is evaluated by substitution into eq. (6). This yields a simplified equation in terms of $A$ and $\Theta$ which, along with eq. (5), can be solved for $A$. The asymptotic amplification, $A$, is found to be

$$
A=\left(\frac{1}{\sin ^{2} 2 \pi f p+\left(\cos 2 \pi f p-\exp \left(-p / \tau_{\mathrm{r}}\right)\right)^{2}}\right)^{\frac{1}{2}}
$$

where the fundamental frequency of oscillation, $f$, is

$$
f=\omega / 2 \pi \text {. }
$$


The amplification is a periodic function of the number of cycles, $f p$, in a pulsation period and a function of the ratio $p / \tau_{\mathrm{r}}$. When the pulse period is an integral multiple of the fundamental period of oscillation, i.e. $f p=N_{\mathrm{c}}$, the amplification is a maximum, given by

$$
A_{\text {max }}=\frac{1}{1-\exp \left(-p / \tau_{\mathrm{r}}\right)} \text {. }
$$

For typical values of $p / \tau_{\mathrm{r}}$, eq. (10) can be approximated by

$$
A_{\max } \approx \tau_{\mathrm{r}} / p
$$

Fig. 1, reproduced from Randles' paper, shows the dependence of the amplification, $A$, on $f p$ for $0.02 \leqslant p / \tau_{\mathrm{r}} \leqslant 0.2$. Two things should be noticed from this figure. First, except very near the resonance, the amplification, which may be less than unity, is independent of $p / \tau_{\mathrm{r}}$. Thus, the phase of the oscillations determines whether or not there will be significant amplification. Second, the quantity $p / \tau_{\mathrm{r}}$ determines the magnitude of the amplification near the resonance. For the values of $p / \tau_{\mathrm{r}}$ considered here, the change in the pulsing frequency necessary to change the amplification from half its maximum value on one side of the resonance to half its value on the other side is

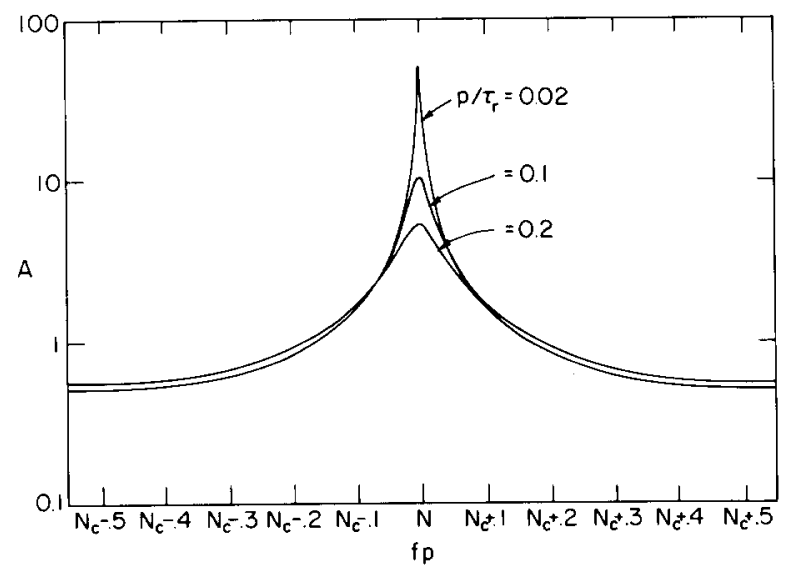

Fig. 1. Asymptotic amplification, $A$, versus the number of cycles per pulsation period, $f p$, for various values of the damping, $p / \tau_{\mathrm{r}}[2]$.

$$
\Delta p \approx \frac{\sqrt{3}}{\pi} \frac{p}{\tau_{\mathrm{r}}} \frac{1}{f}
$$

Finally, from the detailed solution of the amplification as a function of the pulse number, Randles deduced that the time required to reach asymptotic amplification is about four times the relaxation time, $\tau_{\mathrm{r}}$.

\section{Experiment}

In order to see the effect of displacement amplification due to repetitive pulsing experimentally, a large amount of energy must be deposited in the sample at a regular rate of sufficiently high frequency that significant vibrations remain beyond one pulsing period; i.e., $p \ll \tau_{\text {r }}$.

\subsection{Heating method}

The fission heating of a pulsed reactor was simulated electrically, using Joule heating. A high-energy capacitor was discharged quickly through a lowinductance circuit containing the sample. The circuit inductance must be low in order to deposit the energy in the sample in a time short with respect to its mechanical response time.

\subsection{Sample}

In order to approximate the uniform heating which was assumed in the theory, a thin wall, hollow cylinder of type 304 stainless steel was chosen, with a wall thickness of 10 mils, an outside diameter of 0.125 in, and 9 in long. The thin wall is necessary since there is a skin effect in high frequency electrical discharges, where the current tends to concentrate on the outer boundaries of the conductor. In order to support the cylinder, as well as conduct current to and from it, thin brass strips ( 1.5 mills $\times 0.5$ in) were soldered on each end of the sample. The damping effect of these support-electrodes can be shown to be very small. The sample was then supported symmetrically in a current carrying rig such that magnetic forces cancel out. A photograph of a sample mounted in the rig is shown in fig. 2 . 


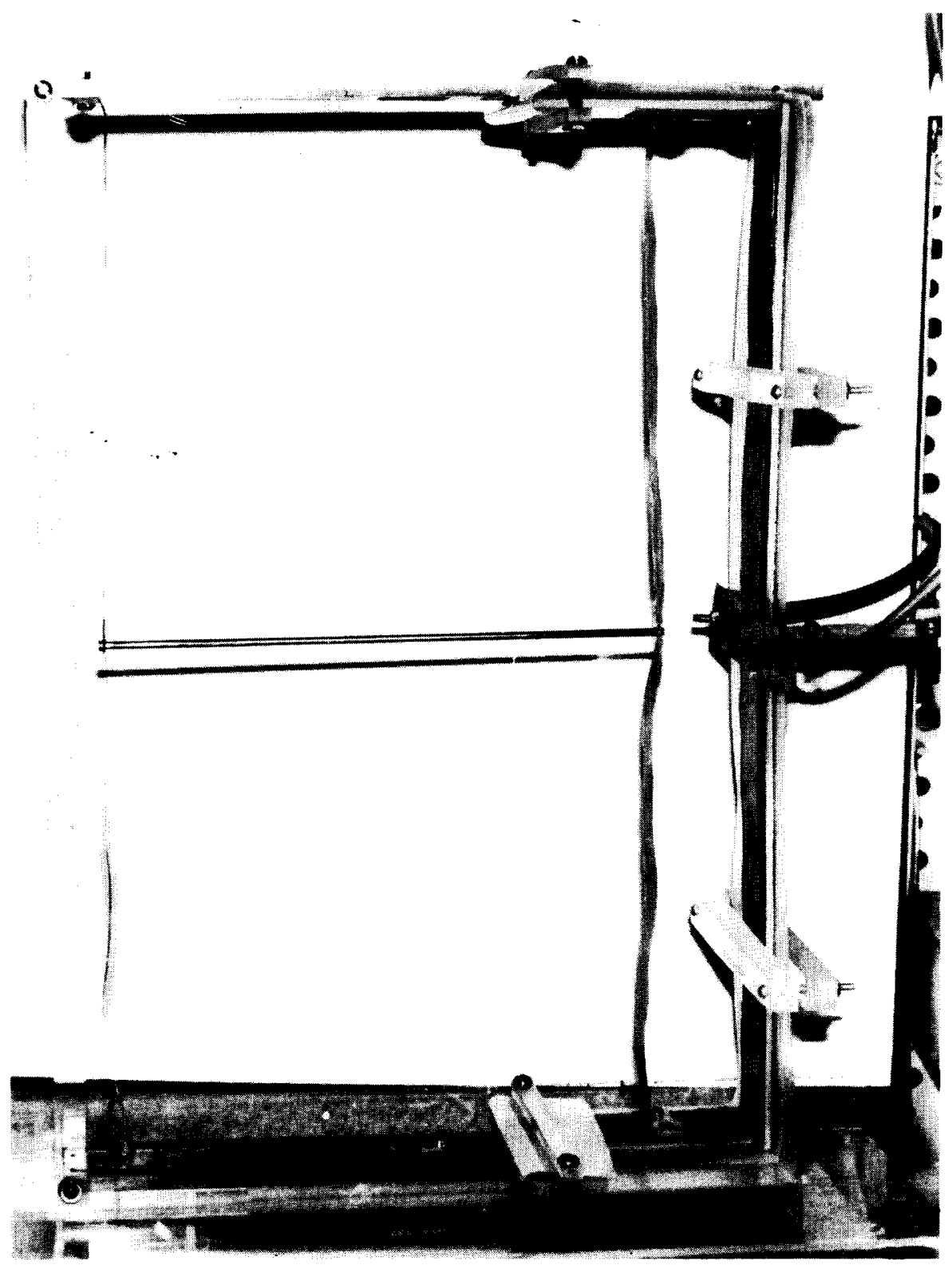

Fig. 2. Sample in current rig.

\subsection{Energy measurement}

In order to determine the total thermal energy, $Q$, deposited in the sample, the current passing through the sample, $i(t)$, and the resistive voltage drop, $V_{\mathrm{R}}(t)$, across it were measured and their product integrated over the discharge time, $t_{0}$. Thus

$$
Q=\int_{0}^{t_{0}} i(t) V_{\mathrm{R}}(t) \mathrm{d} t
$$

The current was measured using a Rogowski Coil [4] which is a toroid winding with the end of the coil 
winding brought back through the turns of the coil so that extraneous magnetic flux through the torus does not thread any portion of the measuring circuit. The alternating current of the discharge circuit was passed through the coil and induced a secondary current which was proportional to the derivative of the discharge circuit current. This was then integrated electrically to yield a signal proportional to the primary current.

The voltage drop across the sample was measured by taking the difference of two Tektronix P-6015 high-voltage probes, one placed at each end of the sample. The subtraction was done electronically, using a Tektronix Type 1A7A High Gain Differential Amplifier. Both the voltage difference and current traces were displayed on a Tektronix Type 551 Dual Beam Oscilloscope.

For an RLC circuit,

$$
\begin{aligned}
& i(t)=C V_{0} \frac{\omega_{\mathrm{c}}^{2}+\alpha_{\mathrm{c}}^{2}}{\omega_{\mathrm{c}}} \exp \left(-\alpha_{\mathrm{c}} t\right) \sin \omega_{\mathrm{c}} t \\
& V_{\mathrm{R}}(t)=v(0) \cot \epsilon \exp \left(-\alpha_{\mathrm{c}} t\right) \sin \omega_{\mathrm{c}} t
\end{aligned}
$$

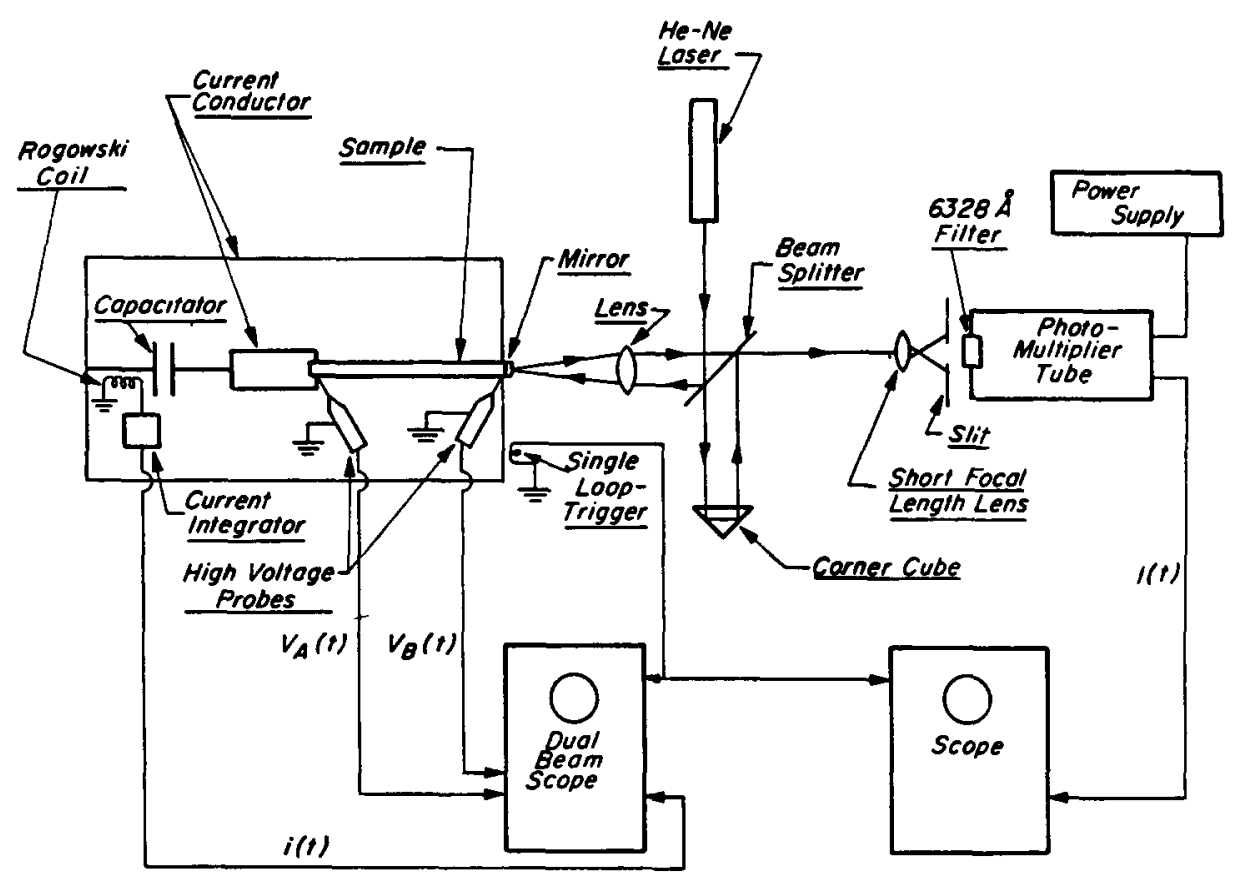

Fig. 3. Schematic diagram of experiment. and $V_{0}$ is the potential across the capacitor, $v(0)$ is the initial potential difference across the sample, and $\epsilon$ is the phase difference between the potential and the current. Thus, the total thermal energy deposited in the sample is given by

$$
\begin{aligned}
Q & =C V_{0} \frac{\omega_{\mathrm{c}}^{2}+\alpha_{\mathrm{c}}^{2}}{\omega_{\mathrm{c}}} v(0) \cot \epsilon \\
& \times \int_{0}^{t_{0}} \exp \left(-2 \alpha_{\mathrm{c}} t\right) \sin ^{2} \omega_{\mathrm{c}} t \mathrm{~d} t .
\end{aligned}
$$

\subsection{Displacement measurement}

The traditional tool for measuring either static or transient displacements is the strain gauge. However, strain gauges were not used in this experiment for two reasons. First, during the capacitor discharge the strain gauge signal was hidden by a large amount of electrical noise. Secondly, for high voltage discharges 
there must be good insulation between the sample and the gauge to prevent arcing; this reduces the accuracy of the strain gauge.

It was desired to use a technique which required only simple data reduction and did not interact with the sample. A technique which satisfies these requirements is the Michelson interferometer using a gas laser light source, developed by Barker and Hollenbach [5].

The measuring system is shown in fig. 3 . The HeNe gas laser, Spectra-Physics Model 132, emits coherent radiation at $6328 \AA$. The beam was split into a reference and a measuring beam. The reference beam was reflected by a fixed corner cube back to the beam splitter. The measuring beam was focused by a lens onto a mirror mounted on the end of the sample. The measuring beam was also reflected back to the beam splitter where it combined with the reference beam to form an interference pattern. Since it was very diffcult to perfectly align the interferometer, localized fringes were used for displacement measurement. These occurred as long as the path difference of the beam splitter to the comer cube and the beam splitter to the mirror was not more than a few millimeters. They appeared as parallel or only slightly curved bands of equal thickness, with the curvature decreasing as the path difference goes to zero [6]. The interference pattern was then enlarged with a short focal length lens, passed through a slit which blocked out all but a single fringe width, and filtered to remove extraneous light sources. The resulting signal was detected by an EMI 9558B photomultiplier tube with $S-20$ spectral response. It was then amplified (Teltronix Type 1A7A high gain amplifier) and displayed on the oscilloscope (Tektronix Type 551). The oscilloscope triggering was done by placing a single wire loop next to the discharge circuit such that a secondary current was induced in the loop. Since the secondary current is proportional to the derivative of the discharge current, a sharp trigger signal was obtained as soon as the capacitor began to discharge.

In addition to the fringe shifts caused by sample vibration, there were additional shifts due to the rigid body motion, or swaying, of the sample. This was due to the normal room vibrations as well as room air currents. These effects were reduced by mounting the interferometer and sample support rig on a four-inch marble slab which in turn sat on four small diameter inner tubes. Also, to reduce air currents, the sample support rig was enclosed by a plexiglass shield.

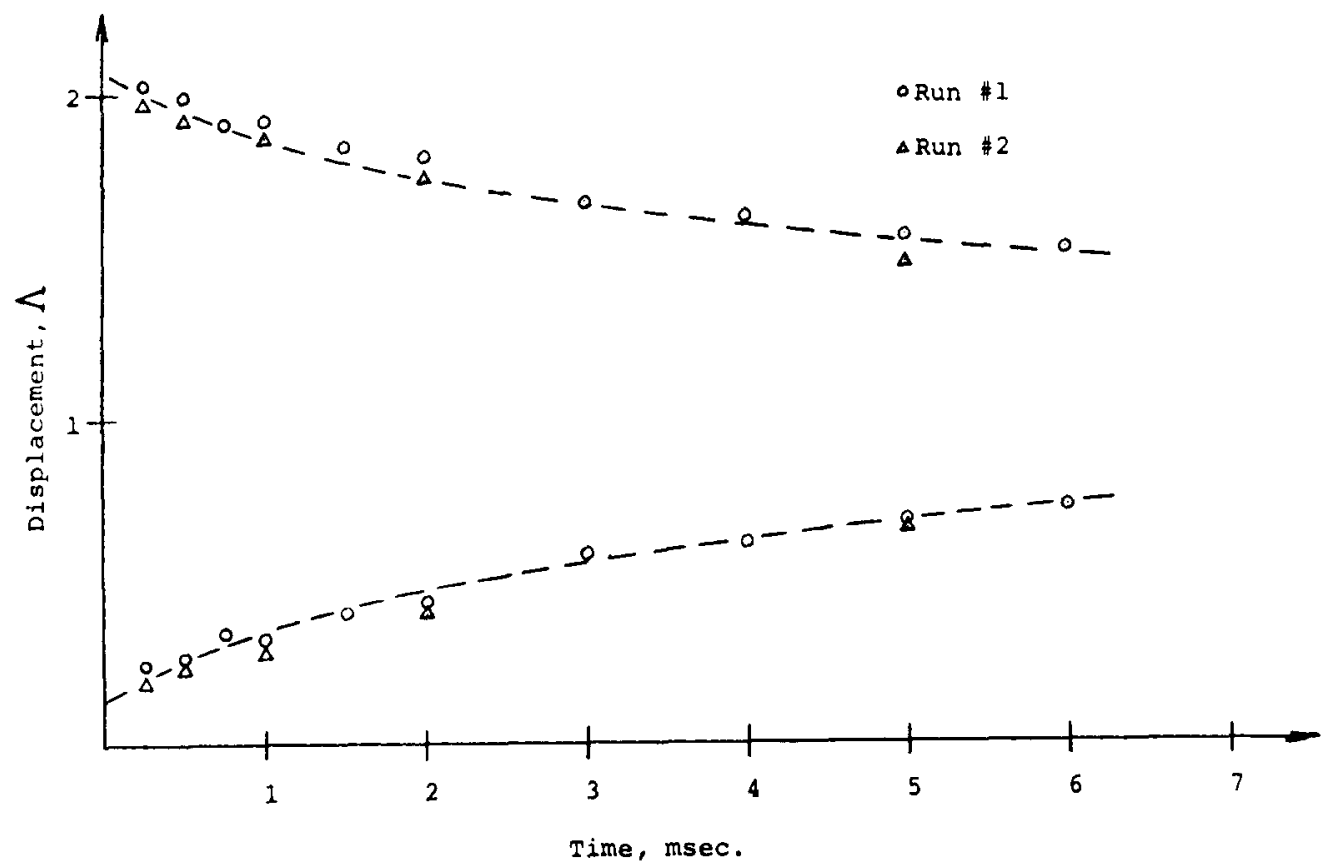

Fig. 4. Displacement versus time for relaxation time measurement. 
As the fringe pattern shifted past the slit, the resulting oscilloscope trace was proportional to the light intensity at the slit. A complete fringe shift, from light to dark to light, corresponded to a displacement of the end of the sample of one-half wavelength, or $3164 \AA$. Thus, displacement was measured by summing the number of fringe shifts, with a resolution of a small fraction of a fringe shift. Most of the measurements in this experiment involved only fractional fringe shifts. In order to compute the displacement corresponding to a fractional fringe shift, the full amplitude must be known. This was done by photographing the fringe shifts due to sample sway at a very low sweep speed with a fast shutter just prior to running the experiment. This gave a single vertical line representing the full fringe shift signal amplitude which was then used for evaluating the fractional fringe shift signals.

When the sample reached a reversal point, there was a pause in the shifts of the fringes, yet the oscilloscope continued to sweep at a constant speed. As a result, the reversal points can be seen quite clearly, and the natural period of vibration thereby determined.

\subsection{Relaxation time measurement}

For computational purposes, the material relaxation time is defined as the $e$-folding time of the envelope of the peak displacements during oscillations due to rapid heating.

An initial measurement of the relaxation time was performed to determine an upper limit on the pulsing period as well as the time required to reach asymptotic amplification. A $14 \mu f$ capacitor was charged to $600 v$ and then discharged through the sample. The resulting oscillations were measured using the laser interferemeter, with the photomultiplier tube output recorded on the oscilloscope. This was repeated for varying oscilloscope sweep delay times so that individual fringes could be seen over a long period of time. The delay was accomplished using a second oscilloscope, Tektronix 317 , to generate a gate pulse of known period. The negative slope at the end of the gate signal was used to trigger the recording oscilloscope.

Since only an estimate of the relaxation time was desired, it was decided to evaluate only a few displacements and assume they were symmetric with respect to the initial expansion, instead of computing each expansion and contraction. The data for two runs are plotted in fig. 4 . From these data, the computed relaxation time is $6 \mathrm{msec}$.

The single discharge can also be used to compute the sample natural frequency of oscillation. Fig. 5 shows the fringe shifts resulting from a $300 v$ discharge from a $14 \mu f$ capacitor. The top trace is the discharge current and the bottom is the fringe shift trace; the sweep speed is $50 \mu \mathrm{sec} /$ division. The reversal points, shown by the arrows, occur every $45 \pm 2 \mu \mathrm{sec}$ apart. This gives a natural frequency of $10.63 \mathrm{kHz}$ $\leqslant f \leqslant 11.62 \mathrm{kHz}$. However, a much more accurate determination of the natural frequency and the relaxation time were made using the repetitive pulsing technique described below.

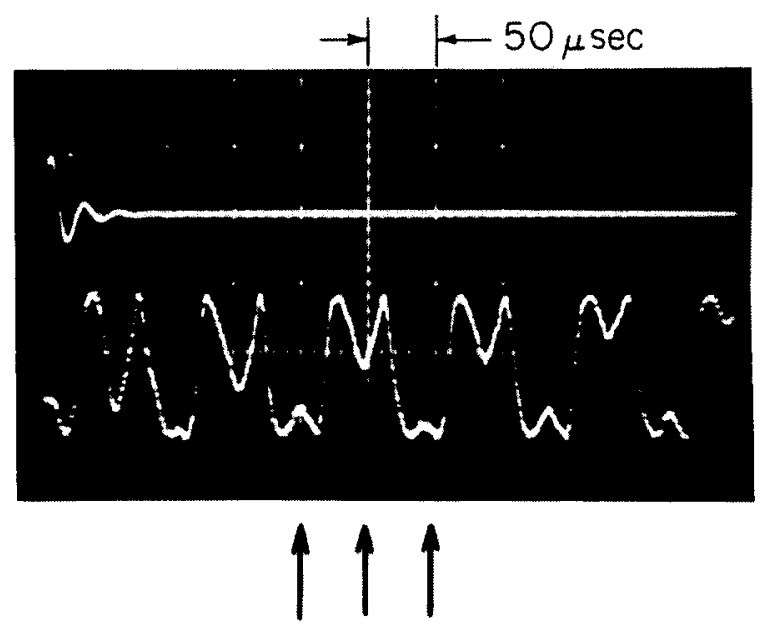

Fig. 5. Natural frequency measurement, Top: discharge current. Bottom: fringe shifts. Arrows indicate reversal points. Sweep speed $=50 \mu \mathrm{sec} /$ division.

\subsection{Experimental restrictions}

Since the material relaxation time is $6 \mathrm{msec}$, it was desired to charge and discharge the capacitor many times within this period. In fact, to obtain the greatest amplification possible, one would like to pulse at the natural frequency.

Furthermore, this amplification only occurs when the pulsing frequency is at or near the natural frequency. From eq. (12), the required resolution of the pulses must be on the order of

$$
\Delta p \approx \frac{\sqrt{3}}{\pi} \frac{\left(90 \times 10^{-6}\right)}{\left(6 \times 10^{-3}\right)} \cdot \frac{1}{\left(1.11 \times 10^{4}\right)}=0.744 \mu \mathrm{sec}
$$


that is, the pulsing frequency must be adjustable to less than $1 \%$.

Thus, the primary requirements of this experiment were rapid energy buidup in the discharge capacitor, a fast switching system to discharge the capacitor, and a high-resolution pulsing technique.

\subsection{Power supply}

At the frequencies of interest it became difficult to deposit enough energy in the sample to measure displacements. Approximately one-half joule was required to record a complete fringe shift. For a pulsing period of about $100 \mu \mathrm{sec}$, the required power, $P$, is

$$
P=\frac{0.5 \text { joule }}{10^{-4} \mathrm{sec}}=5 \mathrm{~kW}
$$

In addition, the charging system time constant must be short enough to charge the capacitor in a time less than the pulsing period. The charging circuit shown in fig. 6 satisfies these requirements. A 12 kilovolt, 1.2 amp power supply (Sorenson, Beta Electric, Type MP12-1.2) was used to charge a $300 \mathrm{pf}$ capacitor through a $10 \mathrm{k} \Omega$ current limiting resistor, yielding a time constant of $30 \mu \mathrm{sec}$.

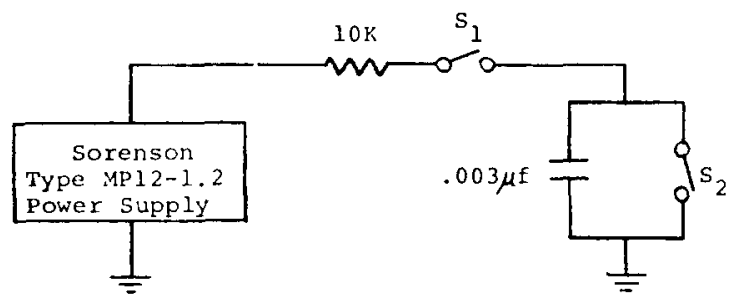

Fig. 6. Repe titive pulsing charging circuit.

\subsection{Discharge technique}

It was required to discharge the capacitor through the sample and open the circuit so that the capacitor could be charged again, within one pulse period, $p$. Furthermore, this switching must be done with voltages on the order of $10 \mathrm{kv}$ and peak currents of approximately $10,000 a$. Electrical switching must be used to acromplish this, since mechanical switches cannot be operated at the required high frequencies.

In view of the above, it was decided to use a spark gap switch to discharge the capacitor. Repetitive pulsing using the static breakdown voltage is not reliable since the statistical time lag for spark formation may be as high as $10 \mu \mathrm{sec}$ [7]. In order to have maximum discharge frequency control, an externally trigge red spark gap was used.

\subsection{Triggering system}

The purpose of the external trigger was to break down the gap and discharge the capacitor at a regular rate. This was accomplished as shown in fig. 7. Assume a potential across the $0.003 \mu f$ discharge capacitor of $10 \mathrm{kV}$. The trigger pin potential is initially $5 \mathrm{kV}$. When the $5 \mathrm{C} 22$ hydrogen thyratron is fired, the pin potential drops to $-5 \mathrm{kV}$. If the gap is set properly, it will immediately break down when the $5 \mathrm{C} 22$ fires. Since the small capacitor can accept very little charge, the trigger pin jumps to $10 \mathrm{kV}$ and, therefore, breaks down the gap between the trigger pin and the low side of the gap.

The $5 \mathrm{C} 22$ has a minimum pulsing period of about $150 \mu \mathrm{sec}$ but is relatively inexpensive and, therefore, it was decided to pulse at twice the natural period. The hydrogen acted as a switch to dump the $50 \mathrm{p} f$ capacitor to ground. The grid required a $2 \mu \mathrm{sec}$ trigger signal of 200-300 V. A C36C high-voltage silicon controlled rectifier (SCR) was used to provide this trigger signal. The $S C R$, in turn, required a gate signal which was provided by an $\mathrm{E}-\mathrm{H}$ Model 133 pulse generator. In order to furnish the desired pulsing period resolution, an additional ten turn precision potentiometer was placed in series with the frequency control.

\subsection{Spark gap extinction}

With the extreme over-vol tage conditions provided by the trigger circuit, the spark gap arc was established within $1 \mu \mathrm{sec}$ and shorted the discharge circuit. Current continued to flow across the gap as long as the gap potential was large enough to maintain the arc. The flow was interrupted by a blast of air which lengthened the arc by contact motion of the air until more potential was required to maintain the arc than was available [8].

Fig. 8 is a photograph of the triggered spark gap switch. The electrodes were one inch diameter, chrome plated, brass hemispheres; the trigger pin was $30 \mathrm{mil}$ diame ter tungsten wire. Air at 120 psi was blown through the gap using a $1 / 16$ inch nozzle placed $3 / 4$ inch from the arc. The nozzle was mounted on a two- 


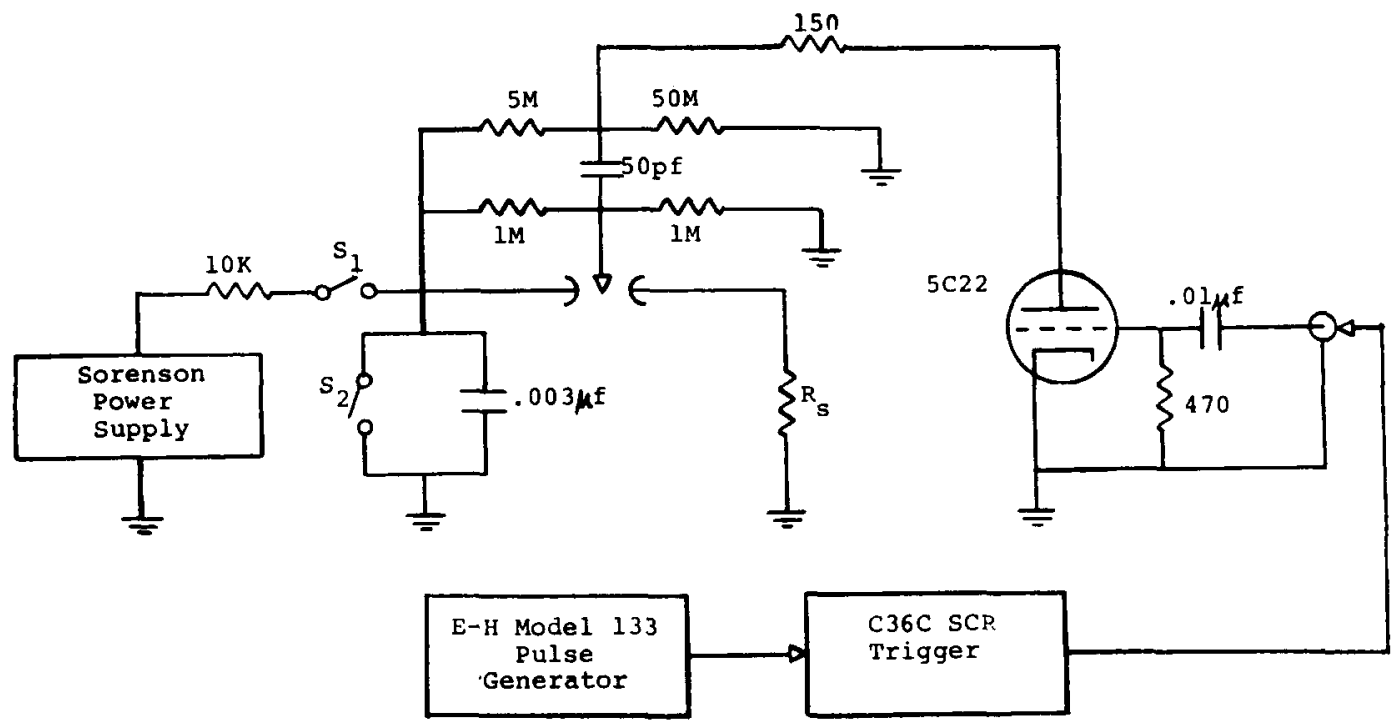

Fig. 7. Complete repetitive pulsing circuit.

stage translation table for fine position control because it was found that the arc extinction time is very sensitive to the blast position; the best results occurred when the blast was centered on the tip of the trigger pin. Experience has shown that for a $10 \mathrm{kV}$ charging voltage, the optimum gap setting is for a $12 \mathrm{kV}$ static breakdown, corresponding to $4 \mathrm{~mm}$ between the electrodes. Fig. 9. is a typical trace of the voltage on the high side (capacitor side) of the gap. At the discharge there was a high frequency oscillation of the voltage at the circuit ringing frequency followed by a conduction period of approximately $50 \mu \mathrm{sec}$ until the arc was quenched and the potential starts to build up again.

\subsection{Amplification measurement}

The desired quantity to be measured is the asymptotic amplification, $A$, of the longitudinal vibration displacements due to repetitive pulsing at an integer multiple of the natural period with respect to the displacement due to a single pulse; that is

$$
\mathrm{A}=\frac{\text { asymptotic longitudinal displacement }}{\text { single pulse displacement }}=\frac{u_{\infty}}{u_{1}}
$$

The first step was to find the proper pulsing period. Since the sample natural period of vibration was ap- proximately $90 \mu \mathrm{sec}$ and the hydrogen thyratron pulse period was limited to about $150 \mu \mathrm{sec}$, a pulse period of twice the natural period was sought. From the relaxation time measurement, the asymptotic displacements occurred after about 24 msec (i.e. four times $\tau_{\mathrm{r}}$ ).

In order to find the resonance, the displacement fringe shifts were observed as a function of the pulse period. The observations were done visually until it was determined that the resonance was found. At this point, photographic recordings of the fringe shift signal and spark gap discharge potential were made, the latter being used to compute the discharge period. The four representative sets of traces in fig. 10 show the fringe shift signal variation with the pulsing period. These traces show the high frequency vibration fringe shift signal superimposed on a much lower frequency fringe shift signal due to the sample rigid body motion. The total sway displacement in one vibration period was approximately 1 percent of the measured maximum asymptotic amplification and, therefore, was ignored in computing the vibration displacement.

From eq. (8) and eq. (21), the asymptotic amplified longitudinal displacement is

$u_{\infty}=u_{1}\left[\frac{}{\sin ^{2} 2 \pi f p+\left(\cos 2 \pi f p-\exp \left(-p / \tau_{\mathrm{r}}\right)\right)^{2}}\right]^{\frac{1}{2}}$ 


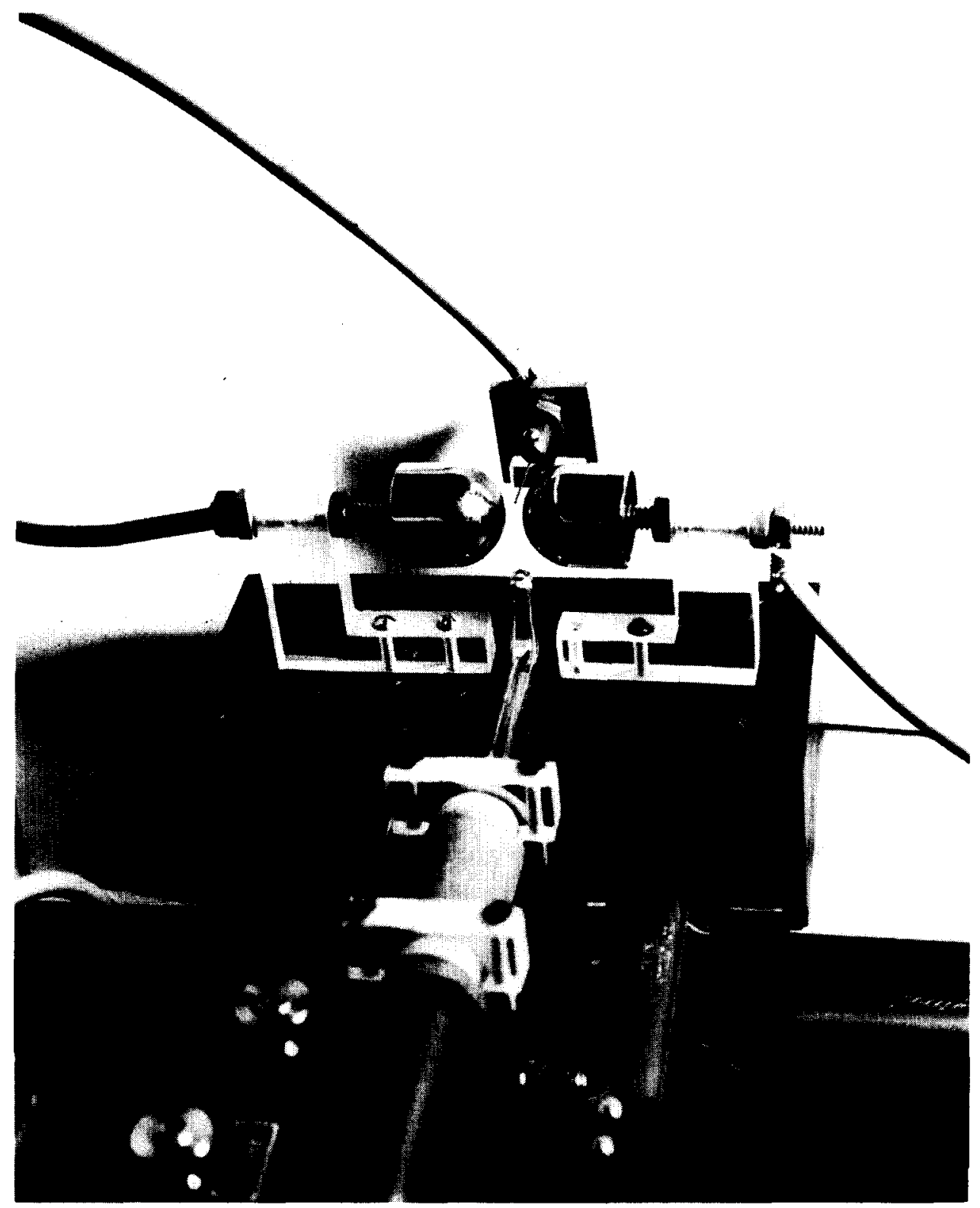

Fig. 8. Spark gap switch.

Fig. 11 is a plot of the measured asymptotic displacements, in terms of fractions of $\Lambda$, for varying pulsing periods. The smooth curve is a least squares fit of eq. (22) through the data points. The least squares analysis gives a natural period of $93.5 \mu \mathrm{sec}$ and a relaxation time of $4.75 \mathrm{msec}$.

An independent measurement of the displacement due to a single pulse was then made. Fig. 12 shows a typical trace of the initial displacement fringe shifts at a pulse period of $187 \mu \mathrm{sec}$ (the vertical line represents the full amplitude signal). It is obvious that the initial displacement is very small, due to the small energy input per pulse, but quickly begins to be amplified with succeeding pulses. The 


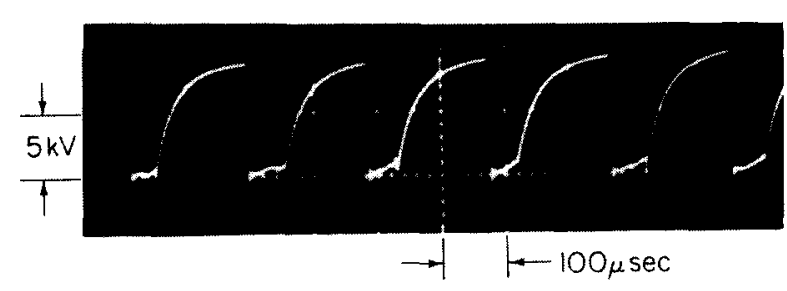

Fig. 9. Potential at high side of spark gap. Vertical: $5 \mathrm{kV} /$ division. Sweep speed $=100 \mu \mathrm{sec} /$ division.

displacement due to the initial pulses increases linearly and, therefore, the expansion displacement due to a single pulse was computed by fitting a least squares regression line to the expansion displacements which occur for the first few pulses following the initial pulse and evaluating the resulting displacement at the first pulse. This was done for four different runs and the best initial displacement was found to be $0.0204 \Lambda$.

\subsection{Results}

The circuit parameters for the discharge circuit were measured as described in section 3.3. Fig. 13 shows the current and voltage drop signals. The circuit parameters, computed using eq. (16) and (17), are shown in table 1 . From fig. $13 \mathrm{~b}$, the phase difference, $\epsilon$, was $32.7^{\circ}$ and the initial voltage, $v(0)$, was $210 \mathrm{~V}$. An upper limit on the energy input per pulse was found by letting $t_{0}$ of eq. (18) approach infinity. The resulting maximum

Table 1

$\begin{array}{ll}\text { Resistance, } R & 3.185 \Omega \\ \text { Inductance, } L & 2.56 \mu \mathrm{H} \\ \text { Capacitance, } C & 0.003 \mu \mathrm{F} \\ \text { Ringing frequency, } f_{\mathrm{c}} & 1.82 \mathrm{MHz}\end{array}$

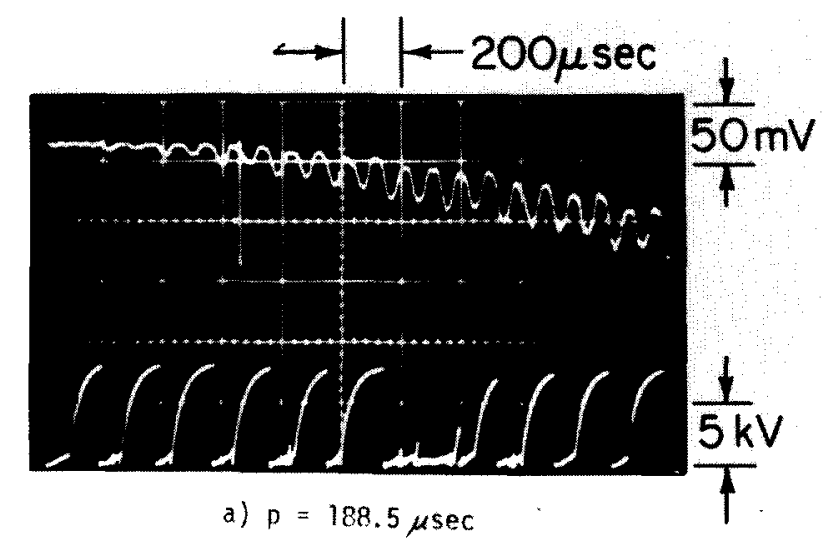

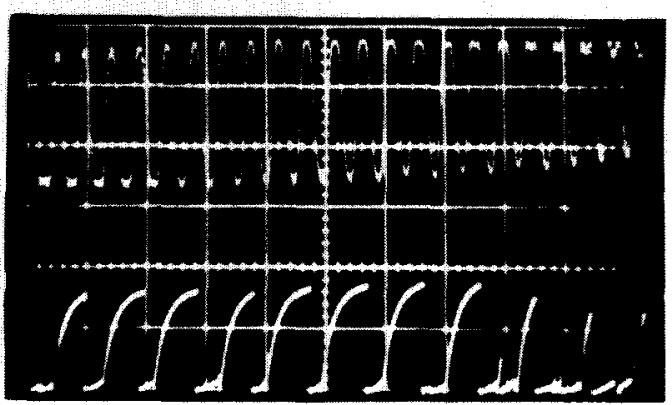

c) $D=19.7 .3 \mathrm{ksec}$

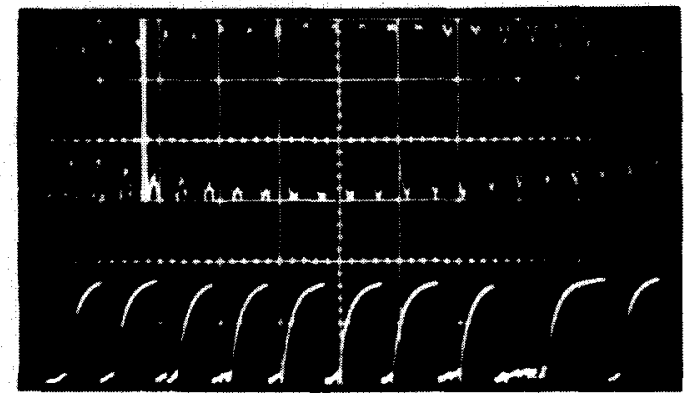

d) $p=187,45 \mathrm{ec}$

Fig. 10. Fringe shift signal variation with pulse period, $p$. Top: fringe shift signal, $50 \mathrm{mV} /$ division. Bottom: spark gap discharge, $50 \mathrm{kV} /$ division. Sweep speed: $200 \mu \mathrm{sec} /$ division. a) $p=188.5 \mu \mathrm{sec}$; b) $p=188 \mu \mathrm{sec}$; c) $p=187.3 \mu \mathrm{sec} ;$ d) $p=187 \mu \mathrm{sec}$. 


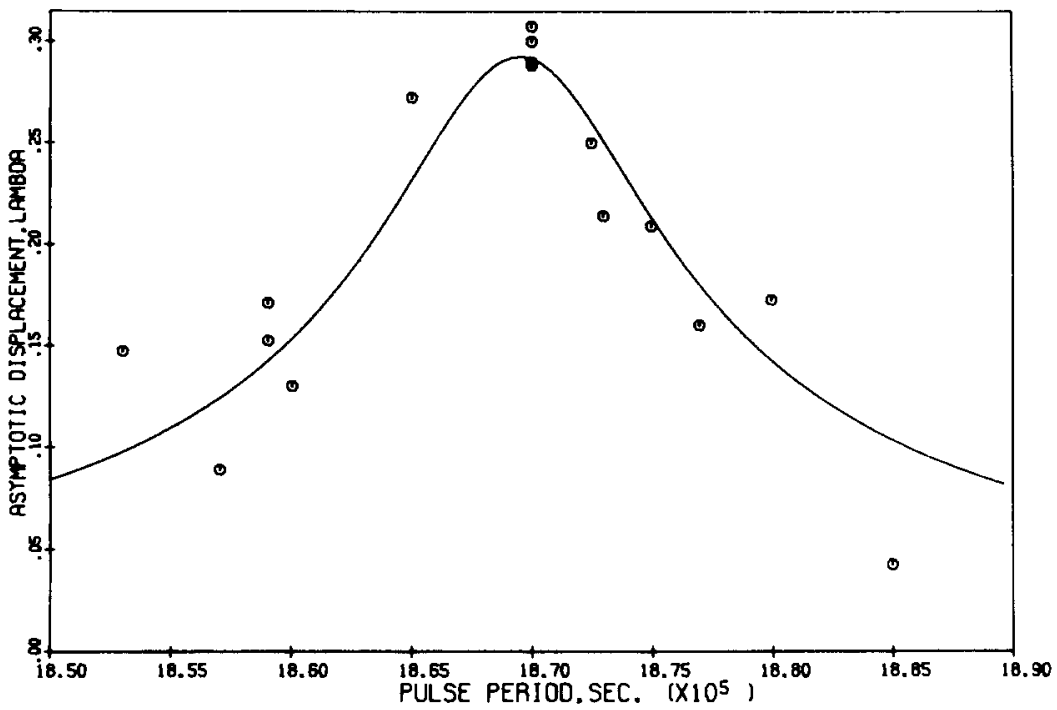

Fig. 11. Asymptotic displacement versus pulse period.

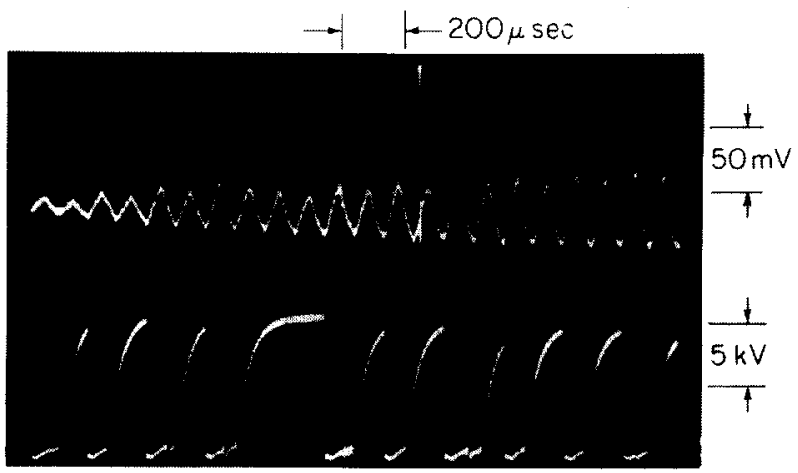

Fig. 12. Initial displacement fringe shifts. Top: fringe shift signal, $50 \mathrm{mV} /$ division. Bottom: spark gap discharge, $50 \mathrm{kV} / \mathrm{division}$. Sweep speed $=200 \mu \mathrm{sec} /$ division.

energy per pulse is $0.045 \mathrm{~J}$. This is one-third of the total energy stored in the $0.003 \mu \mathrm{F}$ capacitor.

The skin depth for type 304 stainless steel at 1.82 $\mathrm{MHz}$ is 12.2 mils. Thus, the sample wall thickness of 10 mils is sufficient to satisfy the assumption of uniform internal heating.

The average maximum asymptotic displacement was found to be $0.2925 \Lambda$, while the best initial displacement value was $0.0204 \Lambda$. Thus, the measured maximum amplification for this system is

$$
A_{\max }=\frac{0.2925 \Lambda}{0.0204 \Lambda}=14.33 \text {. }
$$

\section{Comparison of experimental results with theory}

The theoretical predicted maximum amplification, $A_{\text {max }}$, given by eq. (11) is

$$
A_{\max } \approx \frac{4.75 \times 10^{-3}}{1.87 \times 10^{-4}}=25.4,
$$

where the relaxation time used was that determined from the least squares fit. The experimentally measured maximum amplification was about $60 \%$ of the theoretical value. If the true resonance frequency was not found in the above measurements, it is doubtful that the resonance amplification exceeds the measured 

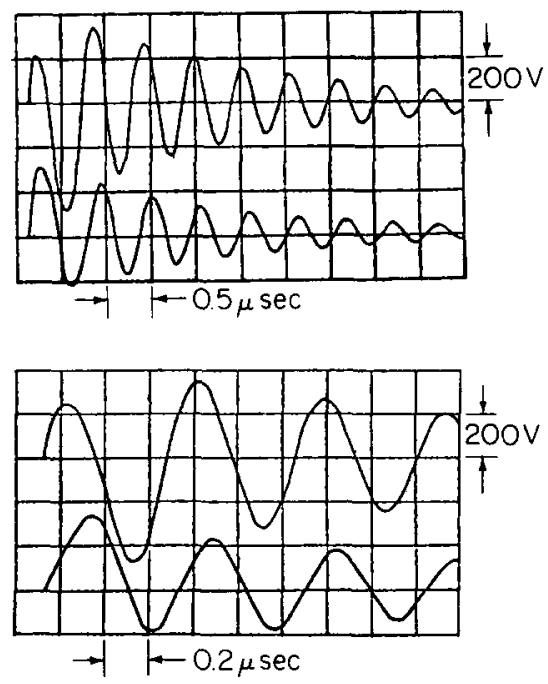

Fig. 13. Voltage drop and current traces for amplitude amplification experiment; a) Top: voltage drop, $20 \mathrm{~V} /$ division. Bottom: current. Sweep speed $=0.5 \mu \mathrm{sec} /$ division; b) Top: voltage drop, $200 \mathrm{~V} /$ division. Bottom: current. Sweep speed $=0.2 \mu \mathrm{sec} /$ division

value by very much, since observations of the fringe shift signal were made with frequency increments of about one-tenth of expected half width of the resonance peak.

The theory assumes an instantaneous step change in temperature which is not possible in practice. The slower temperature rise obtained experimentally will further reduce the maximum amplification since the vibrational energy due to the inertial effect will be diminished for the slower heating rate.

Although the theory assumes a constant relaxation time, this may not acutally be the case. As the displacements increase, the deviation from a purely elastic system increases and, as a result, there may be some change in the relaxation properties of the material. Fichter [9] points out that this can occur even for extremely small strains and that the internal damping is generally a function of the amplitude of the displacement.

\section{Conclusions}

A technique has been developed for rapidly heating a test sample at repetition rates on the order of $5-6$ $\mathrm{kHz}$. The technique makes use of joule heating by discharging a capacitor into a low inductance circuit containing the sample, with the discharge controlled by an externally triggered spark gap switch.

It has been shown that, at the proper pulsing frequency, significant amplification of the vibrational displacements can occur. The longitudinal displacements of a stainless steel hollow cylinder have been measured using a laser interferometer. The measured amplification has been found to be approximately $60 \%$ of the predicted value.

As pointed out by Randles [2], the predicted amplification is for repetitive pulsing with a constant energy input per pulse. In the actual operation of a repetitively pulsed reactor, the start up will be at low energy and gradually build up to the desired value. Thus, there will be sufficient time to determine if the pulsing frequency is going to cause large displacements. During operation, however, it is possible that large amplification could take place if significant deformations occur in the fuel such that the natural frequency is altered.

This experiment is really another form of the resonance technique for measuring internal damping [10]. In that method, the sample is forced to oscillate by some external driver and the resulting strain is measured as a function of the induced frequency. A resonance peak will occur when the induced frequency is the same as the natural frequency of the test sample. The width of this resonance is related to the internal damping of the material, increasing in width as the internal damping increases. Although the primary purpose of this experiment was to measure the maximum amplification under repetitive pulsing, the apparatus described above could be used as a materials test facility for investigating either the internal damping or the low stress, high cycle fatigue strength of various materials. It could also be used to measure the natural frequency and sound speed of a given sample.

\section{Nomenclature}

$A \quad=$ asymptotic amplification factor,

$A_{\max }=$ maximum asymptotic amplification factor,

$A_{m+1}=$ amplification factor for $(m+1)$ st pulse,

$a \quad=$ acoustic velocity $[\mathrm{ft} / \mathrm{sec}]$, 


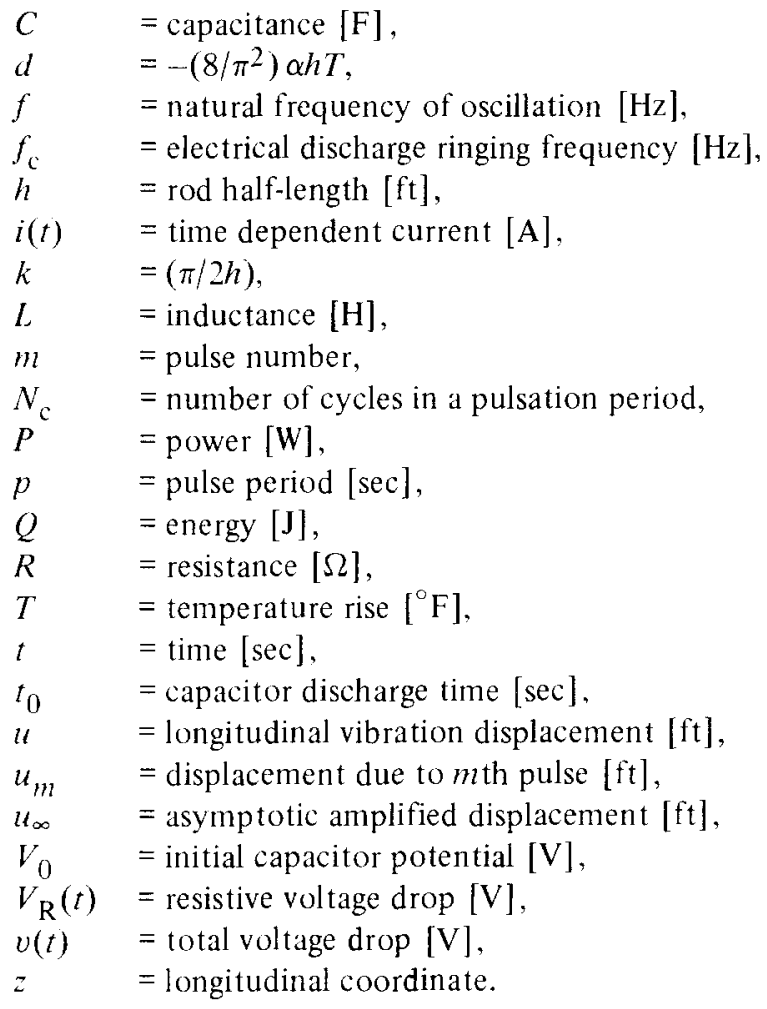

\section{Greek letters}

$\alpha \quad=$ linear thermal expansion coefficient $\left[1^{\circ} \mathrm{F}\right]$,

$\epsilon \quad=$ phase angle between resistive voltage drop and current [rad],

$\Theta \quad=$ asymptotic displacement phase shift [rad],

$\Theta_{m+1}=$ phase shift due to $(m+1)$ st pulse [rad],
$\Lambda \quad=$ laser light wavelength $[\mathrm{ft}]$,

$\tau_{n} \quad=n$th mode relaxation time [sec],

$\tau_{\mathrm{r}} \quad=$ fundamental mode relaxation time [sec],

$\omega \quad=\pi a / 2 h$.

\section{References}

[1] David Burgreen, "Thermoelastic Dynamics of Rods, Thin Shells and Solid Spheres", Nucl. Sci. Eng., 12 (1962) 203.

[2] J. Randles, "Amplification of Vibrations Due to the Repetition of Thermal Shock in a Pulsed Fast Reactor", EUR-4060e (1968).

[3] J. Randles, "Feedback Due to Elastic Waves and Doppler Coefficient during the Excursions of a Pulsed Fast Reactor", J. Nucl. Energy, A/B, 20 (1966) 1.

[4] Richard H. Huddlestone and Stanley L. Leonard, Plasma Diagnostic Techniques, Academic Press, New York (1965).

[5] L.M. Barker and R.E. Hollenbach, "Interferometer Technique for Measuring the Dynamic Mechanical Properties of Materials", Rev. Sci. Instr., 36, 11 (1965) 1617.

[6] Francis A. Jenkins and Harvey E. White, fundamentals of Optics, McGraw-Hill, New York (1957).

[7] L. Marton, ed., Methods of Experimental Physics: Vol. 2, Electronic Methods, by F. Bleuler and R.O. Haxby, Academic Press, New York (1964).

[8] James Dillon Cobine, Gaseous Conductors, Dover, New York (1958).

19) R. Fichter, "Damping in Metals", UKAEA Information Series 14 (D) (1960).

[10] H. Kolsky, Stress Waves in Solids, Dover, New York (1963). 